Revue de droit comparé du travail et de la sécurité sociale

$2 \mid 2020$

La vie personnelle du salarié

\title{
Le respect de la vie personnelle sur le temps et lieu de travail : l'inspection des dossiers informatiques du salarié
}

Sébastien Ranc

\section{(2) OpenEdition}

Journals

Édition électronique

URL : https://journals.openedition.org/rdctss/1118

DOI : $10.4000 /$ rdctss. 1118

ISSN : 2262-9815

Éditeur

Centre de droit comparé du travail et de la sécurité sociale

Édition imprimée

Date de publication : 1 juin 2020

Pagination : 94-101

ISSN : $2117-4350$

Référence électronique

Sébastien Ranc, "Le respect de la vie personnelle sur le temps et lieu de travail : l'inspection des dossiers informatiques du salarié », Revue de droit comparé du travail et de la sécurité sociale [En ligne], 2 | 2020, mis en ligne le 01 novembre 2021, consulté le 11 novembre 2021. URL : http:// journals.openedition.org/rdctss/1118; DOI : https://doi.org/10.4000/rdctss.1118

\section{(c) (i) (3)}

Revue de droit comparé du travail et de la sécurité sociale est mise à disposition selon les termes de la Licence Creative Commons Attribution - Pas d'Utilisation Commerciale - Pas de Modification 4.0 International. 


\title{
LE RESPECT DE LA VIE PERSONNELLE SUR LE TEMPS ET LIEU DE TRAVAIL : L'INSPECTION DES DOSSIERS INFORMATIQUES DU SALARIÉ ${ }^{1}$
}

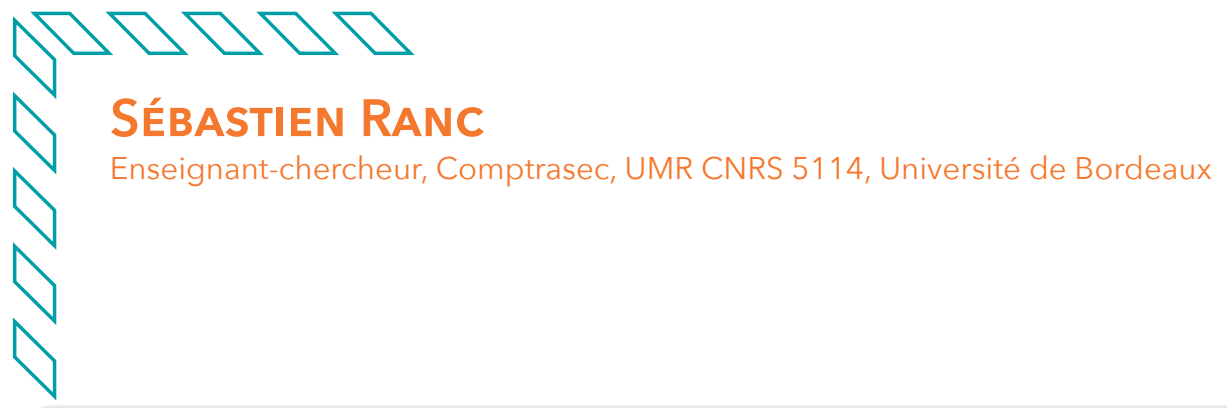

\section{RÉSUMÉ}

Le droit français du travail consacre un droit à une vie personnelle au temps et lieu de travail. En matière d'inspection des dossiers du salarié, le droit français repose sur la distinction relative à la nature du dossier. L'employeur dispose d'un pouvoir de contrôle uniquement à l'égard des dossiers professionnels. II ne peut pas, en principe, accéder aux dossiers identifiés comme personnels. Là est la consécration d'une vie personnelle au temps et lieu de travail. Mais, au fur et à mesure que la frontière devient de plus en plus floue entre vies personnelle et professionnelle - notamment avec le développement des technologies d'information et de communication - il semble que le pouvoir de contrôle de l'employeur empiète de plus en plus sur la vie personnelle du salarié. Cette assertion transparaît en matière de contrôle des documents ou des fichiers informatiques : plus le champ d'application du caractère de professionnalité s'élargit, plus celui du caractère personnel se réduit.

Mots-clés : Vie personnelle, Vie professionnelle, dossier informatique, pouvoir de contrôle de l'employeur

\begin{abstract}
French labour law devoted to a right to a personal life at the time and at the workplace. In terms of inspecting employee files, French law is based on the distinction relating to the nature of the file. The employer has control only over professional records. In principle, he cannot access the files identified as personal. There is the consecration of a personal life at the time and at the workplace. But, as the line between personal and professional lives becomes increasingly blurred - especially with the development of information and communication technologies - it seems that the employer's power of control is encroaching on more in addition to the personal life of the employee. This transparent assertion regarding the control of documents or computer files: the more the field of application of the character of professionalism widens, the more that of the personal character.
\end{abstract}

Key words: Personal Life, Professional Life, IT File, Power of Control of the Employer

1 Cet article est issu d'une intervention lors d'une rencontre du Groupe d'Etude Franco-Allemand sur le Contentieux du Travail (GEFACT), tenue le 19 octobre 2019 à Toulouse. 


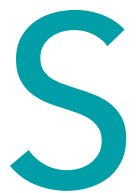

i la vie personnelle s'exerce en principe en dehors du travail, le droit français reconnaît l'existence d'un droit à une vie personnelle au temps et lieu de travail. En effet, la vie personnelle ne se réduit pas à la vie extraprofessionnelle. Cette vie personnelle, qui regroupe toutes les libertés du citoyen (notamment le droit à la vie privée) et les droits fondamentaux, subsiste et persiste pendant la vie professionnelle.

Par exemple, une utilisation à des fins personnelles des outils mis à la disposition par l'employeur est en principe tolérée, si elle reste raisonnable et ne dégénère pas en abus. Ainsi, ont été considérées comme abusives des connexions internet à des fins privées durant 4 heures ${ }^{2}$, ou à plus de 10000 reprises en un mois ${ }^{3}$.

La vie personnelle du salarié au temps et lieu de travail est protégée par l'article L. 1121-1 du Code du travail, selon lequel «nul ne peut apporter aux droits des personnes et aux libertés individuelles et collectives de restrictions qui ne seraient pas justifiées par la nature de la tâche à accomplir ni proportionnées au but recherché ».

Autrement dit, la vie personnelle du salarié au temps et lieu de travail peut certes être réduite, mais en aucun elle ne peut être supprimée. La restriction devra être justifiée par la nature de la tâche à accomplir et proportionnée au but recherché.

La vie personnelle du salarié au temps et lieu de travail peut donc faire l'objet d'un contrôle par l'employeur ${ }^{4}$ et l'inspection des dossiers du salarié en est une illustration. La jurisprudence s'est construite progressivement à partir de la question du contrôle par l'employeur des fichiers informatiques, des mails ou encore de l'historique informatique, figurant pour chacun d'eux sur l'ordinateur professionnel. Un tel contrôle est évidemment impossible à l'encontre du matériel dont la propriété relève de celle du salarié.

À l'origine, la jurisprudence a pu être très protectrice de la vie personnelle du salarié au temps et au lieu de travail. Elle avait posé un principe d'interdiction de consultation par l'employeur des fichiers informatiques personnels du salarié.

Dans le célèbre arrêt dit Nikon ${ }^{5}$, après avoir visé l'article 8 de la Convention européenne de sauvegarde des droits de l'homme et des libertés fondamentales, l'article 9 du Code civil, l'article 9 du Code de procédure civile et l'ancien article L. 120-2 du Code du travail - devenu l'article L. 1121-1 - la Chambre sociale de la Cour de cassation a décidé

2 Soc., 18 mars $2009, n^{\circ} 07-44.247$.

3 Soc., 26 février 2013, n¹1-27.372.

4 L'introduction dans la messagerie professionnelle peut parfois provenir d'un collègue de travail. Voir not. CE, 10 juillet 2019, n²08644 : SSL 2019, n²1872, p. 5, concl. F. Dieu.

5 Soc., 2 octobre 2001, n99-42.942. 
que « le salarié a droit, même au temps et lieu de travail, au respect de l'intimité de sa vie privée ; que celle-ci implique en particulier le secret des correspondance ; que l'employeur ne peut dès lors sans violation de cette liberté fondamentale prendre connaissance des messages personnels émis par le salarié et reçus par lui grâce à un outil informatique mis à sa disposition pour son travail et ceci même au cas où l'employeur aurait interdit une utilisation non professionnelle de l'ordinateur ».

Ce principe d'interdiction de consultation par l'employeur des messages informatiques personnels du salarié a été tempéré en matière de dossiers informatiques.

L'intensité du pouvoir de contrôle de l'employeur varie alors selon la nature des dossiers du salarié. Si les dossiers sont professionnels, l'employeur dispose d'un vaste pouvoir de contrôle (I). En revanche, si les dossiers sont personnels, le pouvoir contrôle de l'employeur est limité par la vie personnelle du salarié (II).

\section{I - UN POUVOIR DE CONTRÔLE ILLIMITÉ À L'ÉGARD DES DOSSIERS PROFESSIONNELS}

Les dossiers et fichiers créés par le salarié grâce à l'outil informatique professionnel mis à sa disposition par l'employeur sont présumés être professionnels (A). L'employeur dispose à leur égard d'un pouvoir quasiment illimité de contrôle (C). Seuls sont a priori protégées les documents identifiés comme personnels par le salarié (B).

\section{A - LE CHAMP EXTRÊMEMENT LARGE DE LA PRÉSOMPTION SIMPLE DE PROFESSIONNALITÉ}

Selon la Chambre sociale de la Cour de cassation, « les dossiers et fichiers créés par un salarié grâce à l'outil informatique mis à sa disposition par son employeur pour l'exécution de son contrat de travail sont présumés, sauf si le salarié les identifiait comme personnels, avoir un caractère professionnel, de sorte que l'employeur pouvait y avoir accès hors de sa présence $"^{6}$. Autrement dit, les outils mis à la disposition du salarié pour l'exécution de son travail ont à priori une destination professionnelle.

Le champ d'application de cette présomption de professionnalité est très -voire trop - large. La jurisprudence applique évidemment cette présomption simple aux documents sous forme de papier ${ }^{7}$. Sont aussi concernés les connexions internet effectuées pendant le temps de travail à partir de l'ordinateur professionnel ${ }^{8}$, les mails adressés ${ }^{9}$ ou reçus ${ }^{10}$ par le salarié avec l'ordinateur professionnel.

\footnotetext{
Soc., 18 octobre 2006, n04-48.025.

Soc., 18 octobre 2006, $n^{\circ} 04-47.400 ; 4$ juillet 2012, $n^{\circ} 11-12.330$.

Soc., 9 juillet $2008, n^{\circ} 06-45.800 ; 9$ février $2010, n^{\circ} 08-45.253$.

Soc., 30 mai 2007, n05-43.102; 15 décembre 2010, n08-42.486.

10 Soc., 18 octobre $2011, n^{\circ} 10-26.782$.
} 
Il existe même une sorte de "présomption de professionnalité par destination ". À partir du moment où un objet personnel est relié à un objet mis à la disposition par l'employeur pour l'exécution du travail, l'objet personnel - ou plutôt son contenu - devient professionnel.

Par exemple, " une clé USB, dès lors qu'elle est connectée à un outil informatique mis à la disposition du salarié par l'employeur pour l'exécution du contrat de travail, est présumée utilisée à des fins professionnelles. En conséquence, les dossiers et les fichiers non identifiés comme personnels qu'elle contient, sont présumés avoir un caractère professionnel de sorte que l'employeur peut y avoir accès hors la présence du salarié « ${ }^{11}$.

Par un même raisonnement, les SMS envoyés ou reçus au moyen d'un téléphone professionnel12, ou les mails émanant de la messagerie personnelle du salarié, mais intégrés dans le disque dur de l'ordinateur professionnel ${ }^{13}$, sont présumés être professionnels.

Cette dernière "présomption de professionnalité par destination » peut sembler critiquable dans la mesure où la messagerie personnelle distincte de la messagerie professionnelle devrait être en principe protégée par le « donjon de la vie personnelle ${ }^{14}$, à l'intérieur duquel se trouvent la vie privée et, par conséquent, le secret des correspondances ${ }^{15}$.

C'est ainsi que, au nom d'un tel secret, un arrêt récent de la Cour de cassation protège des messages électroniques issus d'une messagerie instantanée (MSN Messenger) installée sur un ordinateur professionnel, mais distincte de la messagerie professionnelle ${ }^{16}$.

Autrement dit, s'agissant de l'utilisation de la messagerie électronique, la jurisprudence est quelque peu différente de celle relative aux dossiers informatiques, du fait de l'application du secret des correspondances qui conduit à accorder une protection plus importante aux salariés.

\section{B - L'IDENTIFICATION DU CARACTÈRE PERSONNEL}

Seuls les documents informatiques identifiés comme personnels sont protégés. On est ici dans une démarche "proactive » du salarié, ce dernier devant lui-même protéger ses fichiers ou documents en les identifiant comme " personnels».

11 Soc., 12 février 2013, n¹1-28.649.

12 Com., 10 février 2015, n¹3-14.779.

13 Soc., 19 juin 2013, $n^{\circ} 12-12.138$.

14 « De même que le donjon était la partie la mieux protégée du château fort, la vie privée apparaît comme le cœur de la vie personnelle, l'élément le mieux protégé, le plus résistant de la vie personnelle : I'intimité de la vie privée »: Ph. Waquet, Y. Struillou et L. Pécaut-Rivolier, Pouvoir du chef d'entreprise et libertés du salarié. Du salarié-citoyen au citoyen-salarié, éd. Liaisons, 2014, numéro spéc., p. 178.

15 Soc., 26 janvier 2016, n¹4-15.360. En outre, l'atteinte au secret des correspondances est pénalement répréhensible (C. pén., art. 226-15).

16 Soc., 23 octobre 2019, nº17-28.448. 
À cet égard, la Cour de cassation se montre très - voire une fois de plus trop - rigoureuse. Si la dénomination «mes documents » ne permet pas d'identifier un fichier comme étant personnel ${ }^{17}$, ou que la dénomination "données personnelles » choisie pour le disque dur ne confère pas un caractère personnel à l'intégralité des données qu'il contient ${ }^{18}$, on reste perplexe sur le fait que les initiales $" \mathrm{JM} »^{19}$, ou le prénom du salarié, ne suffise pas à caractériser le caractère personnel du fichier informatique ${ }^{20}$.

\section{C - UN POUVOIR DE CONTRÔLE ILLIMITÉ DES DOCUMENTS PROFESSIONNELS}

À partir du moment où les dossiers sont de nature professionnelle, l'employeur dispose d'un accès illimité à ces dossiers et n'a pas à en informer le salarié ni, a fortiori, à lui en demander l'autorisation.

Toutefois, le " contrôle surprise » est permis. Ce contrôle peut même s'effectuer en l'absence du salarié. Ce dernier ne dispose d'aucune garantie procédurale.

En revanche, s'il s'avère que le contenu des documents de nature professionnelle relève de la vie privée du salarié, l'employeur ne pourra pas se servir de ce contenu personnel comme moyen probatoire devant le juge ${ }^{21}$, pas plus qu'il ne pourra l'utiliser pour prendre une mesure disciplinaire ${ }^{22}$.

Cette dernière solution n'est que la conséquence du fait qu'un fait de la vie personnelle du salarié ne peut justifier le prononcé d'une sanction disciplinaire à son encontre. Seul un contenu en "rapport avec l'activité professionnelle ${ }^{23}$ peut conduire à une sanction disciplinaire.

Il faut donc distinguer l'accès au dossier professionnel de leur utilisation par l'employeur. Le contrôle de l'accès au dossier par l'employeur est en quelques sorte «léger », puisque le juge se contente de vérifier la dénomination du dossier.

Tandis que le contrôle de l'utilisation du dossier par l'employeur à des fins probatoires ou sanctionnatrices est plus «lourd », dans la mesure où le juge va contrôler que le contenu du dossier est en « rapport avec l'activité professionnelle » et qu'il n'a pas été porté atteinte à la vie privée du salarié.

17 Soc., 10 mai 2012, n¹1-13.884. Précisons que la dénomination «mes documents " se trouve par défaut sur tous les ordinateurs. II n'y a donc aucune démarche " proactive » de la part du salarié d'identifier un tel fichier comme personnel.

18 Soc., 4 juillet 2012, $n^{\circ} 11-12.502$.

19 Soc., 21 octobre 2009, $n^{\circ} 07-43.877$.

20 Soc., 8 décembre 2009, n08-44.840.

21 Soc., 18 octobre $2011, n^{\circ} 10-25.706$.

22 Soc., 5 juillet 2011, $n^{\circ} 10-17.284$.

23 Soc., 28 septembre 2011, n¹0-16.995; 2 février 2011, n09-72.313. 
Si le contrôle par l'employeur des documents présumés professionnels se révèle être sans limite, en revanche celui des documents personnels du salarié semble a priori délimité.

En réalité, cette protection de la vie personnelle au temps et lieu de travail à travers les dossiers informatiques ne résiste pas à l'analyse.

\section{II - UN POUVOIR DE CONTRÔLE LIMITÉ À L'ÉGARD DES DOSSIERS PERSONNELS}

Dans une affaire où un salarié avait été licencié à la suite de la découverte de photos érotiques dans un tiroir de son bureau, puis dans un fichier informatique intitulé " perso", la Cour de cassation avait décidé, au visa des articles 8 de la Convention européenne de sauvegarde des droits de l'homme et des libertés fondamentales, 9 du Code civil, 9 du Code de procédure civile et L. 120-2 [actuel L. 1121-1] du Code du travail, que « sauf risque ou évènement particulier, l'employeur ne peut ouvrir les fichiers identifiés par le salarié comme personnels contenus sur le disque dur de l'ordinateur mis à sa disposition qu'en présence de ce dernier ou celui-ci dûment appelé » ${ }^{24}$.

\section{A - LE RESPECT DU PRINCIPE DU CONTRADICTOIRE}

Pour que l'employeur puisse accéder aux dossiers personnels du salarié, il doit le faire en présence du salarié ou, au minimum, l'avoir informé d'un tel contrôle. Le " contrôle surprise » est en principe interdit.

Néanmoins, l'employeur n'a pas à recueillir l'accord du salarié. Si le salarié a été prévenu, mais qu'il refuse d'y participer, l'employeur peut tout de même procéder à l'ouverture des fichiers en question en veillant à la présence d'un huissier et/ou des représentants du personnel. Il s'agit là d'une reprise des garanties procédurales issues d'une jurisprudence en matière de contrôle des casiers individuels des salariés ${ }^{25}$.

\section{B - LA POSSIBILITÉ D'UN « CONTRÔLE SURPRISE » DES DONNÉES PERSONNELLES DU SALARIÉ}

En cas de "risque ou évènement particulier ", l'employeur n'a plus à respecter le principe du contradictoire. Il s'agit encore une fois d'une modalité du contrôle empruntée à la jurisprudence en matière de contrôle des casiers individuels des salariés ${ }^{26}$. On retombe ici dans un pouvoir de contrôle illimité des documents personnels du salarié à l'instar du contrôle des documents professionnels.

24 Soc., 17 mai 2005, $n^{\circ}$ 03-40.017.

25 Soc., 11 décembre 2001, n 99-43.030.

26 Soc., 11 décembre 2001 , ibid. 
Toute la difficulté réside dans la définition du « risque ou de l'évènement particulier ». En l'espèce, la découverte de photos érotiques dans un tiroir ne constitue pas une telle circonstance ${ }^{27}$. On dispose d'un exemple jurisprudentiel en matière de contrôle visuel des sacs personnels à l'entrée de l'entreprise : une série d'attentats et des alertes à la bombe ont pu constituer des " circonstances exceptionnelles et des exigences de sécurité ${ }^{28}$.

En outre, un virus, une attaque informatique, la crainte de voir se développer des réseaux pénalement répréhensibles, une concurrence déloyale grave ${ }^{29}$, ou encore un dénigrement de la société par voie informatique, pourraient constituer un "risque ou un évènement particulier » qui permettent à l'employeur de contrôler par surprise les documents personnels du salarié.

\section{C - SUR L'ASSIMILATION DU MONDE VIRTUEL AU MONDE PHYSIQUE}

Le dossier informatique du $21^{\text {e }}$ siècle correspond à ce que le casier individuel du salarié était au $20^{\circ}$ siècle, en l'occurrence un espace de la vie personnelle au temps et lieu de travail ${ }^{30}$. La jurisprudence a ainsi assimilé le régime du dossier informatique à celui du casier personnel.

Pour rappel, le régime juridique applicable au contrôle du casier du salarié est déterminé par la réunion de trois conditions à respecter :

1. la nécessité d'une clause expresse du règlement intérieur prévoyant le principe et l'encadrement des fouilles ${ }^{31}$;

2. le contrôle doit être justifié par les nécessités de la santé ou de la sécurité dans l'entreprise ${ }^{32}$;

3. I'information préalable des salariés ${ }^{33}$.

27 Soc., 17 mai 2005, op. cit.

28 Soc., 3 avril 2001, n98-45.818. En l'espèce, il s'agissait d'un contrôle visuel des sacs, et non d'une fouille. La fouille d'un sac personnel dans l'entreprise nécessite de recueillir l'accord du salarié, après l'avoir informé de la possibilité de s'opposer à une telle fouille et d'exiger la présence d'un témoin. Soc., 11 février 2009, n07-42.068. Cette protection renforcée se comprend au regard de la nature du sac qui est un bien dont la propriété est celle du salarié.

29 Soc., 10 juin 2008, n06-19.229 : ouverture de fichiers non personnels au motif que l'employeur avait des « raisons légitimes et sérieuses de craindre que l'ordinateur mis à la disposition de la salariée a été utilisé pour favoriser des actes de concurrence déloyale ».

30 F. Favennec-Héry, "Conditions d'ouverture par l'employeur des fichiers identifiés comme personnels du salarié », JCP S 2005, p. 1031.

31 Soc., 11 décembre 2001, n99-43.030.

32 CE, 8 juillet 1988 : D. 1990, p. 134, note D. Chelle etX. Prétot. Adde CE 12 novembre 1990, n94778. En l'espèce, le contrôle avait été admis pour une entreprise utilisant des matériaux dangereux et des substances explosives et pour laquelle des vols seraient susceptibles d'engendrer des risques d'une particulière gravité.

33 Soc., 15 avril 2008, n06-45.902. Auparavant, la jurisprudence imposait la présence du salarié pour l'ouverture de son casier individuel : Soc., 11 décembre 2001, n99-43.030. 


\section{FRANCE}

Le casier individuel apparait ainsi mieux protégé que les fichiers informatiques explicitement affichés comme personnels. La distinction dans la protection accordée au casier individuel et les fichiers personnels pourrait s'expliquer par l'utilisation du bien en question par le salarié.

Si le casier individuel et l'ordinateur professionnel au sein duquel se trouve les fichiers personnels relèvent de la propriété de l'employeur, le casier est pour sa part uniquement destiné à un usage personnel (dépôt d'affaires personnelles), tandis que sur l'ordinateur professionnel se côtoient des utilisations personnelle et professionnelle.

En conclusion, l'assimilation de la protection accordée à la vie personnelle du monde virtuel à celle du monde physique reste incomplète. Cette remarque vaut autant pour l'assimilation du dossier informatique, que pour celle du mail à la correspondance papier.

Ces solutions semblent peu compatibles avec l'existence d'une vie personnelle au temps et lieu de travail. 